\title{
Electromagnetic vs. Plane Wave Models for Superdirective 2D Adaptive HF Receive Antenna Performance Assessment
}

\author{
Geoffrey San Antonio \\ US Naval Research Laboratory \\ Washington, DC 20375 USA
}

\author{
Yuri I. Abramovich \\ WR Systems \\ Fairfax, VA 22030 USA
}

\author{
Gordon J. Frazer \\ NSI Division, DSTO \\ Edinburgh, SA, AUS
}

\author{
Charles Williams \\ Gadzooks Pty. Ltd. \\ Melbourne, VC, AUS
}

\begin{abstract}
Index Terms-HF OTHR, 2D Arrays, electromagnetic model, superdirectivity
\end{abstract}

\section{INTRODUCTION}

In a number of recent papers [1], [2], [3], [4], [5], twodimensional (2D) antenna arrays with regular rectangular or hexagonal element spacing structures and inter-element spacing $d$ smaller than one-half the radar wavelength $\lambda$

$$
d<\lambda / 2
$$

have been analyzed for HF operations in the presence of strong external noise, typical for night time skywave propagation conditions [6], [7]. It has been demonstrated that for typical 2D external noise distributions, localized within the "noise ring" below the critical elevation angle, optimal beamforming for such antenna arrays provides essential signal-to-external noise ratio (SENR) gains with respect to conventional beamforming. More specifically, it has been demonstrated that for near grazing elevation angles, these SENR gains are primarily achieved due to the superdirective properties of the optimal (or adaptive) beamformer which shifts a significant part of the conventional main beam into the so-call "invisible" spatial angle region [8], [9], [12]. In the case of surface wave over-thehorizon radar (OTHR) which always has its receive main-beam steered to $0^{\circ}$ elevation angle with respect to the horizon, this property of the optimal beamformer in oversampled 2D arrays is very important. In contrast, for beamsteering elevation angles close or above the critical elevation angle, as per some HF line-of-sight applications, very substantial SENR gains can be demonstrated by the optimal (adaptive) beamformer due to the re-distribution of the beampattern volume away from the "external noise ring," in the noise free area at high elevation angles. Yet, within the elevation sector of 10-20 degrees, the optimum performance in these arrays is very close to performance of conventional beamforming and is up to $4 \mathrm{~dB}$ worse than for the grazing (elevation=0 $\mathrm{deg}$ ) angle. For sky-wave operations this degradation is essential, despite the fact that in general, higher elevation angles correspond to a shorter distances with a smaller propagation loss and therefore higher SNR on a specified target cross-section. Therefore, when available physical space permits, more appropriate 2D geometries could be proposed to avoid this degradation; a practical example of such an array is introduced in our companion paper [13]. Since superdirective properties in these arrays are also exploited, performance analysis of 2D oversampled (dense and sparse) arrays is quite important for sky-wave, surfacewave and line-of-sight applications.

\section{Numerical Electromagnetic Manifold MODELING}

Prior analysis of SENR gains in these arrays has been conducted with ideal plane-wave antenna manifold models which corresponds to the impractical open-circuit loaded antenna model [4], [10]. Naturally, it was always understood that for practical performance evaluation, the accurate electromagnetic (EM) model of such an array erected on a ground with realistic properties and certain ground screen arrangement, should be computed. Moreover, unlike long uniform linear arrays (ULA) with apertures greater than $2-3 \mathrm{~km}$ and the number of antenna elements $N>300$ where only a small number of edge elements are under non-homogeneous EM conditions, in 2D rectangular arrays the non-homogeneous EM conditions should be expected in two dimensions. Since in most designs, the width of a rectangular $2 \mathrm{D}$ array is much smaller than its length, this non-homogeneity is of great concern, since it cannot be controlled by a heavy taper, as per multielement ULA's. These concerns affect the antenna element design as well. Indeed, the more matched and broadband antenna elements are, the larger the external-to-internal noise ratio (EINR) that could be delivered by those elements and therefore potentially higher SENR gains may be provided by the optimal (adaptive) beamformer. On the other hand, matched antenna elements are more coupled as well and if this increased mutual coupling is not accounted for in the actual antenna manifold used in optimal (adaptive) beamforming the SENR degradation caused by the mismatch between the actual antenna manifold and its (plane wave) model, could be very severe as per any superdirective array. Therefore, a comparison of the EM-predicted and plane-wave model derived properties of the optimum (adaptive) beamformer, is needed to address a number of very important questions.

In consideration of the above mentioned concerns, the following set of issues have to be addressed. 
1) Are the potential gain predictions for a typical external noise model derived based upon the accurate EM model of a $2 \mathrm{D}$ array, the same as per the simplistic planewave model? Note, the potential SENR are assessed with EM-predicted antenna manifold vectors used both for external noise and target response representation.

2) How significant are SENR mismatch losses in the optimal (adaptive) beamformer designed for plane-wave target response instead of the accurate EM model prediction?

3) How sensitive are EM manifold predictions with respect to minor variations of the EM parameters, such as for example the ground conductivity (results of rain, snow, etc.)?

4) How accurate is the traditional $N \times N$-variate coupling matrix model with respect to the ground and ground screen contribution to the EM model?

5) How accurate could the antenna calibration procedures be that exploit ground-wave propagated test signals?

These issues are critically important for practical applications of 2D oversampled antenna arrays. In this paper we address some of these issues, while their full analysis will be presented in the subsequent full paper.

In this paper we compute some very basic array performance metrics to evaluate the behavior of specific array configurations, external noise models, and array manifold models. First let us define the standard plane wave manifold vector

$$
\mathbf{a}_{p}(\theta, \phi)=\exp (-j 2 \pi / \lambda \mathbf{X} \mathbf{u}(\theta, \phi))
$$

where $\mathbf{X}$ is the $N \times 3$ matrix of array element positions and $\mathbf{u}(\theta, \phi)$ is the unit vector in the direction $(\theta, \phi)$. In the case of the numerical electromagnetic modeled vector, the array manifold is denoted as $\mathbf{a}_{e m}(\theta, \phi)$. The array spatial covariance matrix due to external noise can be obtained by integration of the external noise model with the array manifold model. We consider three methods for computing the external noise covariance.

$$
\begin{array}{r}
\mathbf{R}_{p-i n t-\text { mod-ext }}=\int_{0}^{\pi / 2} \int_{0}^{2 \pi} f(\theta, \phi) b_{e l-m o d}(\theta, \phi) \times \\
\mathbf{a}_{p}(\theta, \phi) \mathbf{a}_{p}^{H}(\theta, \phi) \sin (\theta) d \theta d \phi \\
\mathbf{R}_{p-f s-m o d-e x t}=\sum_{i=1}^{N_{i}} \sum_{j=1}^{N_{j}} f\left(\theta_{i}, \phi_{j}\right) b_{e l-m o d}\left(\theta_{i}, \phi_{j}\right) \times \\
\mathbf{a}_{p}\left(\theta_{i}, \phi_{j}\right) \mathbf{a}_{p}^{H}\left(\theta_{i}, \phi_{j}\right) \sin \left(\theta_{i}\right) \\
\mathbf{R}_{e m-f s-e x t}=\sum_{i=1}^{N_{i}} \sum_{j=1}^{N_{j}} f\left(\theta_{i}, \phi_{j}\right) \times \\
\mathbf{a}_{e m}\left(\theta_{i}, \phi_{j}\right) \mathbf{a}_{e m}^{H}\left(\theta_{i}, \phi_{j}\right) \sin \left(\theta_{i}\right)
\end{array}
$$

The overall array spatial noise covariance is computed as the sum of external and internal noise, weighted appropriately to achieve a specific per-element external-to-internal noise ratio.

$$
\mathbf{R}=c \mathbf{R}_{e x t}+\mathbf{I}
$$

For simplicity we denote the external plus internal noise covariance for the planar and EM manifold models as $\mathbf{R}_{p}$ and $\mathbf{R}_{e m}$ respectively. The planar manifold covariance model used the integral calculation in (3), however in the simulations is is shown to be equivalent to the finite sum model (4). Our calculations of array gain and mismatch utilize two types of beamforming solutions, the conventional beamformer and the optimal beamformer. These are computed as follows.

$$
\begin{gathered}
\mathbf{w}_{p, c o n v}(\theta, \phi)=\frac{\mathbf{a}_{p}(\theta, \phi)}{\mathbf{a}_{p}^{H}(\theta, \phi) \mathbf{a}_{p}(\theta, \phi)} \\
\mathbf{w}_{p, o p t}(\theta, \phi)=\frac{\mathbf{R}_{p}^{-1} \mathbf{a}_{p}(\theta, \phi)}{\mathbf{a}_{p}^{H}(\theta, \phi) \mathbf{R}_{p}^{-1} \mathbf{a}_{p}(\theta, \phi)} \\
\mathbf{w}_{e m, c o n v}(\theta, \phi)=\frac{\mathbf{a}_{e m}(\theta, \phi)}{\mathbf{a}_{e m}^{H}(\theta, \phi) \mathbf{a}_{e m}(\theta, \phi)} \\
\mathbf{w}_{e m, o p t}(\theta, \phi)=\frac{\mathbf{R}_{e m}^{-1} \mathbf{a}_{e m}(\theta, \phi)}{\mathbf{a}_{e m}^{H}(\theta, \phi) \mathbf{R}_{e m}^{-1} \mathbf{a}_{e m}(\theta, \phi)}
\end{gathered}
$$

These beamforming solutions are used to compute both array signal-to-external noise ratio gains and mismatch losses.

$$
\begin{gathered}
\operatorname{SENR}_{g a i n}(\theta, \phi)=\frac{\left|\mathbf{w}^{H} \mathbf{a}_{e m}(\theta, \phi)\right|^{2}}{\mathbf{w}^{H} \mathbf{R}_{e m} \mathbf{w}} \times \frac{1}{\operatorname{SNR}_{e l m}(\theta, \phi)}(11) \\
\mathrm{ML}_{w h t}(\theta, \phi)=\frac{\left|\mathbf{a}_{p}^{H}(\theta, \phi) \mathbf{a}_{e m}(\theta, \phi)\right|^{2}}{\mathbf{a}_{p}^{H}(\theta, \phi) \mathbf{a}_{p}(\theta, \phi) \mathbf{a}_{e m}^{H}(\theta, \phi) \mathbf{a}_{e m}(\theta, \phi)}(12) \\
\mathrm{ML}_{c l r}(\theta, \phi)=\frac{\left|\mathbf{a}_{p}^{H}(\theta, \phi) \mathbf{R}_{e m}^{-1} \mathbf{a}_{e m}(\theta, \phi)\right|^{2}}{\mathbf{a}_{p}^{H}(\theta, \phi) \mathbf{R}_{e m}^{-1} \mathbf{a}_{p}(\theta, \phi) \mathbf{a}_{e m}^{H}(\theta, \phi) \mathbf{R}_{e m}^{-1} \mathbf{a}_{e m}(\theta, \phi)}
\end{gathered}
$$

Note, in (11) the SENR gain is defined with respect to the signal-to-external noise ratio of the median element in the array.

\section{Simulations}

The EM modeling used in this paper uses the electromagnetic code Numerical Electromagnetic Code (NEC) [11] which is a method-of-moments based computational electromagnetic code. It requires the specification of the following design parameters: antenna array geometry, antenna element, antenna element height, type of ground screen, ground conductivity, frequency, far-field azimuth angles and elevation angles of point sources. For this study we consider a specific antenna array design consisting of a $17 \times 72 \mathrm{D}$ rectangular antenna array with hexagonal element spacing having inter-element spacing $d=11.7856 \mathrm{~m}$. An $h=5.5 \mathrm{~m}$ tall vertical monopole with diameter $\phi=38 \mathrm{~mm}$ was directly connected to a $50 \Omega$ receiver input (no transformer or matching network used). Every element has been provided with 16 equispaced wire radials of length $l=32 \mathrm{ft}$ and diameter $\phi=1.6 \mathrm{~mm}$. For the dry ground conditions considered the antenna array response has been calculated on a grid with angular spacing of $0.5^{\circ}$ for elevation angles $0^{\circ}-90^{\circ}$ and azimuth angles from $0^{\circ}-360^{\circ}$. 
Given this set of antenna array responses, the external noise covariance matrix has been calculated via discrete numerical integration over the upper hemisphere space, weighted in accordance with a specifically considered external noise spatial distribution. These results have been compared with similar results derived using the plane-wave array manifold model and the same external noise distribution. An example of this comparison is shown in figure (1) and (2). Here the antenna array SENR gain with respect to a single (median) antenna element are introduced both for conventional and optimal beamformers for the frequency $f_{c}=8.4849 \mathrm{MHz}$, noise distribution

$$
f(\theta, \phi)=c \sin ^{10}(\phi),
$$

and $\mathrm{EINR}=30 \mathrm{~dB}$.

In the case where the plane-wave array manifold model is combined with the analytic antenna element model $\left(\sin ^{2}(\phi) \cos ^{0.7}(\phi)\right)$ and the analytic noise distribution model given by equation (14), the array external noise covariance has been calculated analytically in [4]. This allows us to asses the accuracy of the finite-sum covariance model approximation applied in the EM manifold model.

To simplify the presentation of the results we specifically examine two extreme azimuthal directions (boresight and endfire). In these directions we examine the antenna array gains as a function of elevation angle. In the first set of figures, (1) and (2), we observe SENR gains calculated for the EM model external noise covariance matrix and antenna manifold, for both the optimum and conventional beamformers calculated for the accurate (EM model) and erroneous (planewave model) target response model. One can see that the conventional beamformer is practically insensitive to antenna manifold mismatch. On the contrary, the optimum beamformer operating in a superdirective mode $\left(\phi \leq 30^{\circ}\right)$, is quite sensitive to the manifold mismatch. Indeed, while the proper (EM model) steering vector used for the optimum beamforming mismatch results in very significant SENR gains, reaching $6-8 \mathrm{~dB}$ at $\phi<5^{\circ}$, and being superior to the conventional beamformer for any steering elevation angle, if the plane-wave model is used we can actually significantly degrade the SENR performance of the "optimal" (adaptive) beamformer compared with even the conventional beamformer. For reference we show the case for the plane-wave based noise covariance in figures (3) and (4).

Another comparison that can be made involves the comparison of the analytic array covariance matrix model versus the finite sum approximation in the case of the plane-wave model (applied to both the noise and target). We observe that granularity of the spatial sampling grid is fine enough to accurately approximate the true covariance with a finitesum approximation. In figures (3) and (4) this approximation accuracy is evident by the SENR line plots (denoted by the suffixes "intcov" and "fscov") that practically overlap. We can then compare how the finite sum plane-wave covariance model compares to the EM based finite sum covariance model. The predicted array SENR gains are very similar in both cases, which indicates that the plane-wave model is useful enough to be used in array performance prediction analysis. This is shown in figures (3) and (4). Another comparison we can make is to adopt a slightly altered element pattern in the plane-wave array model based on the EM model median element pattern, shown in figure (5). This accounts for slight modeling differences between our analytic element model $\left(\sin ^{2}(\phi) \cos ^{0.7}(\phi)\right)$ and the actual embedded element pattern. Little difference $(<0.5 d B)$ in SENR results with this modification.

The detailed analysis demonstrated that the plane-wave model is adequate for a potential SENR performance assessment, but may lead to significant SENR loss if applied directly to an actual antenna array adaptive beamforming scenario. Figures (6), (7), and (8) show the planewave-mismatch loss.

\section{CONCLUSION}

This paper demonstrated how a 2D planar oversampled HF receive array manifold can be modeled by NEC and utilized to asses the performance of conventional and optimal beamforming techniques. It is shown that the full EM array model behaves similar to a planewave manifold model in the presence of strong non-isotropic external noise. Furthermore it is demonstrated that the optimal beamformer solutions computed with a manifold not calibrated to the true EM manifold will exhibit some amount of mismatch loss in directions of strong external noise. In a subsequent full paper, we will comment further on the robustness of the EM model antenna manifold predication to small model perturbations (i.e. changes in ground conditions, element errors, etc.) and on the efficiency of a number antenna calibration techniques that can be used to avoid the SENR losses associated with antenna manifold mismatch.

\section{ACKNOWLEDGMENT}

This work was sponsored by the Office of Naval Research under an NRL 6.1 Base Program

\section{REFERENCES}

[1] Y. I. Abramovich and G. San Antonio, "Regularized Finite-Order FiniteRank Covariance Matrix Approximation for Adaptive Beamforming in Oversampled 2D HF Antenna Arrays," IEEE Radar Conf., Cincinnati, OH May 2014.

[2] Y. I. Abramovich and G. San Antonio, "Over-the-Horizon Radar Potential Signal Parameter Estimation Accuracy in Harsh Sensing Environments," ICASSP, Florence, Italy, May 2014.

[3] G. San Antonio and Y. I. Abramovich, "Oversampled 2D OTHR Receive Array Superdiective SNR Gains," Tri-Service Radar Conf., Springfield, VA, July, 2014

[4] Y. I Abramovich and G. S. San Antonio and G. J. Frazer, "Over-theHorizon Radar Signal-to-External Noise Ratio Improvement in OverSampled Uniform 2D Antenna Arrays: Theoretical Analysis of Superdirective SNR Gains,” IEEE Radar Conf., Ottawa, CA, May 2013.

[5] Y. I Abramovich and G. S. San Antonio, "Aperture Varying Autoregressive Covariance Modeling for 2D Oversampled Receive Arrays," CAMSAP, St. Martin, 15-18 Dec. 2013.

[6] J. M. Headrick and S. J. Anderson, "HF Over-the-Horizon Radar," in Radar Handbook, 3rd ed., M. Skolnik, Ed. New York: McGraw-Hill, 2008, ch. 20.

[7] International Radio Consultive Committee (CCIR) Report 322-3, "Characteristics and Applications of Atmospheric Radio Noise Data," Documents of the XVth Plenary Assembly, Geneva, 1983. 
[8] R. J. Mailloux, Phased Array Antenna Handbook, Boston, MA: Artech House, 2005, 2nd Ed.

[9] H. L. Van Trees, Optimum Array Processing, New York: WileyInterscience, 2002.

[10] Y. I Abramovich and G. S. San Antonio, "Network Theory-Based Generic Model for Oversampled Receive Antenna Arrays," SAM 2012 Conf., Hoboken, New Jersey, 17-22 June 2012.

[11] G. J. Burke and A. J. Poggio, "Numerical Electromagnetic Code (NEC) User's Guide," Lawrence Livermore Laboratory, CA, 1981.

[12] H. Salt, "Practical realization of superdirective arrays," The Radio and Electronic Engineer, vol. 47, no. 4, pp. 143-156, April 1977.

[13] G. J. Frazer, C. G. Williams, Y. I Abramovich, and G. S. San Antonio, "Regular Two-Dimensional Over-Sampled Sparse Receiving Array for Over-the-Horizon Radar," Submitted to IEEE Int. Radar Conf., May 2015.

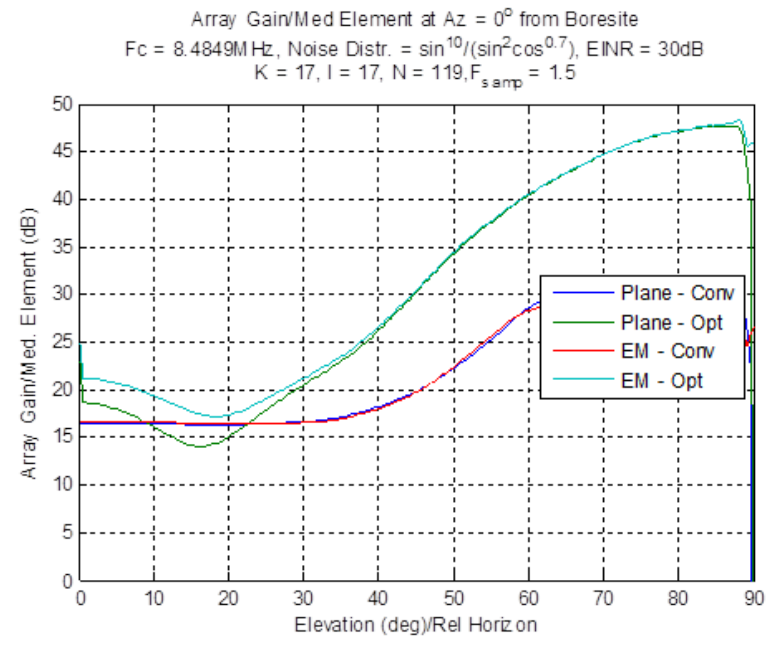

Fig. 1. Boresight SENR gains using an EM model based array spatial noise covariance. Several beamformer techniques shown.

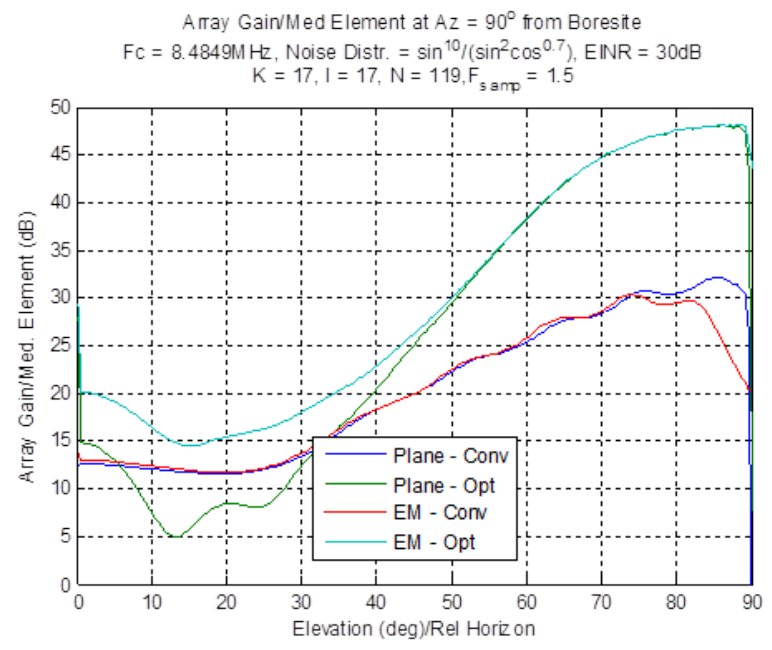

Fig. 2. Endfire SENR gains using an EM model based array spatial noise covariance. Several beamformer techniques shown. 


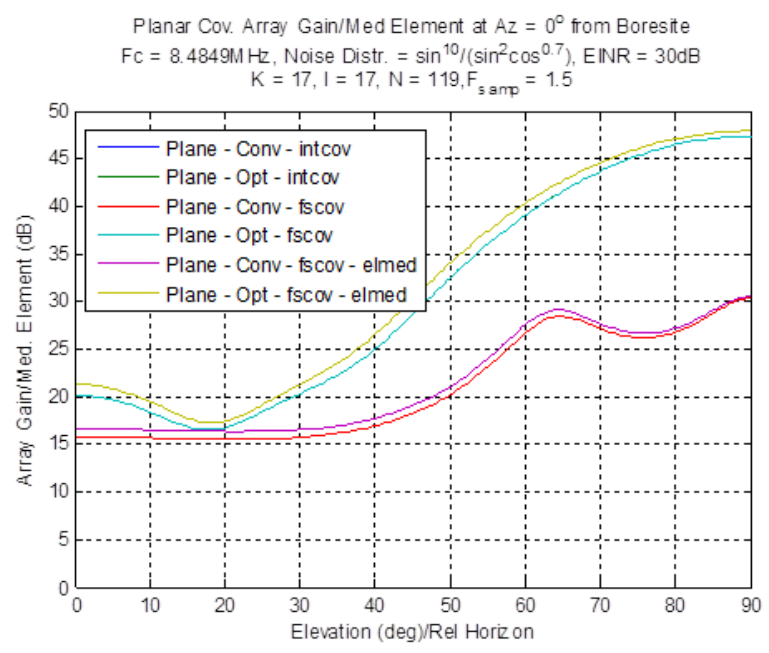

Fig. 3. Boresight SENR gains using a plane-wave model based array spatial noise covariance. Several beamformer schemes and covariance matrix calculation techniques shown.

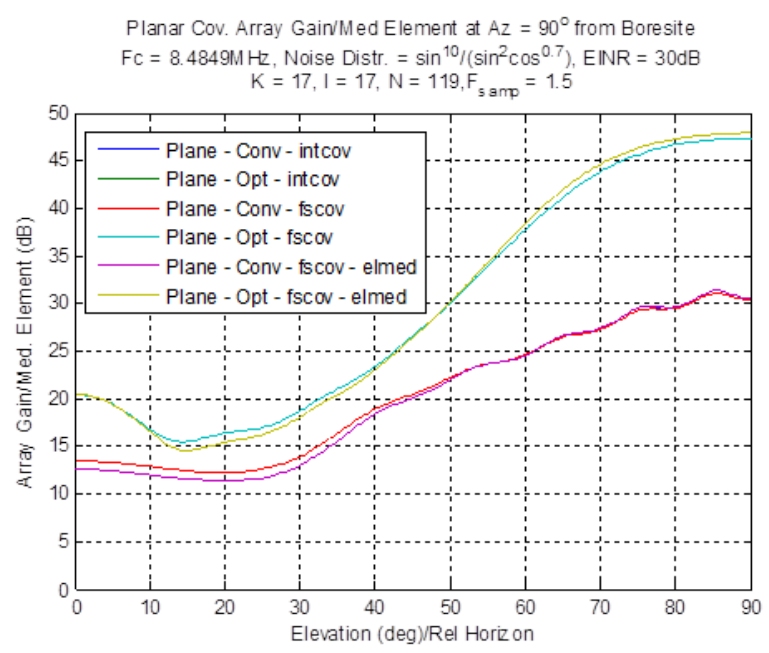

Fig. 4. Endfire SENR gains using a plane-wave model based array spatial noise covariance. Several beamformer schemes and covariance matrix calculation techniques shown.

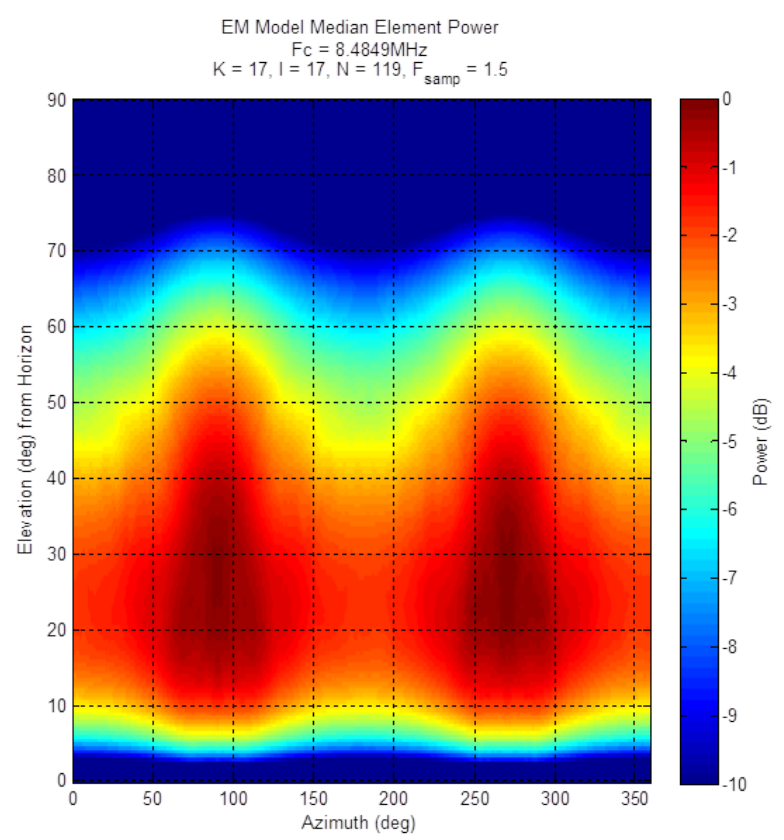

Fig. 5. Median element model derived from EM array manifold model.

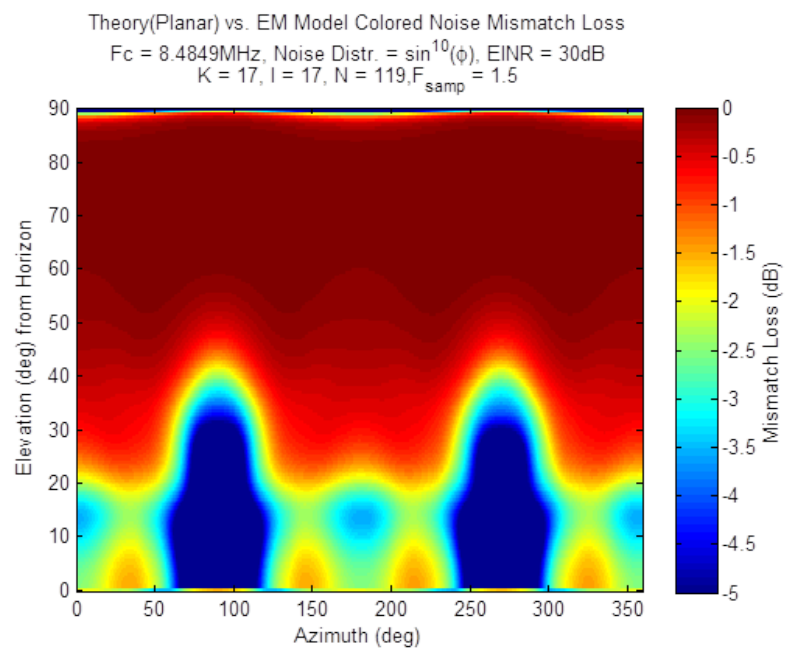

Fig. 6. Mismatch loss incurred by using plane-wave model in the place of true EM model. 


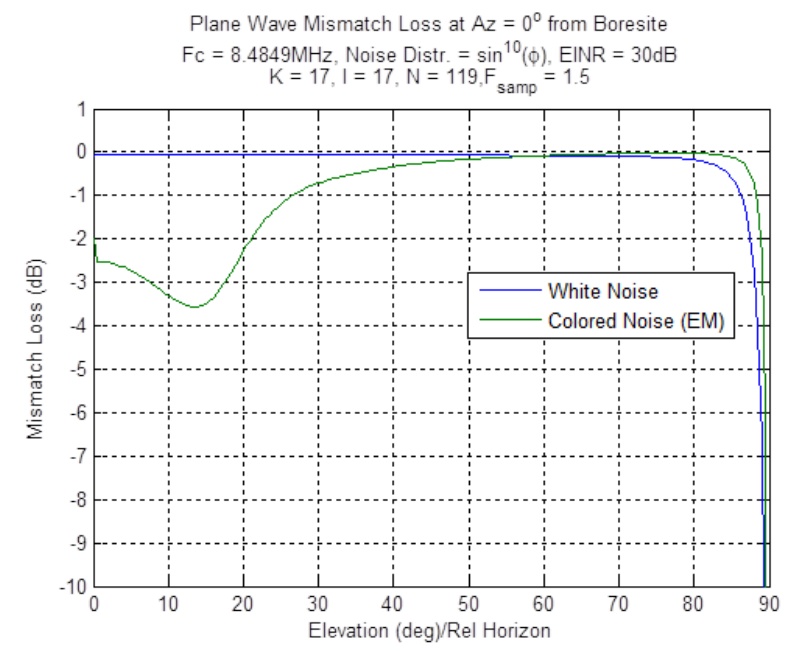

Fig. 7. Mismatch loss (at boresight azimuth) incurred by using plane-wave model in the place of true EM model.

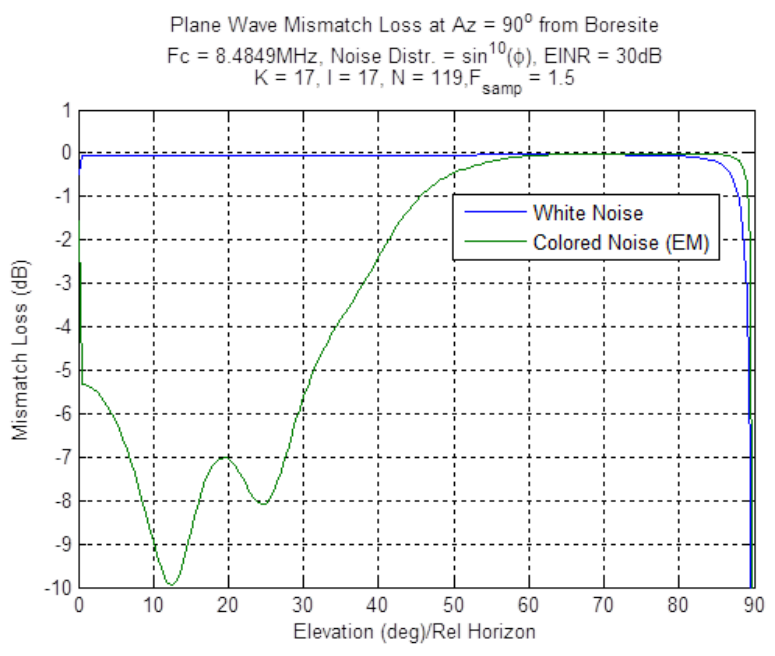

Fig. 8. Mismatch loss (at endfire azimuth) incurred by using plane-wave model in the place of true EM model. 